\title{
Elevated Vascular Level of ortho-Tyrosine Contributes to the Impairment of Insulin-Induced Arterial Relaxation
}

\author{
Authors \\ I. A. Szijártón ${ }^{1}$, 2, 3, 4, G. A. Molnár ${ }^{1}$, E. Mikolás ${ }^{1}$, V. Fisi ${ }^{1}$, J. Cseh ${ }^{1}$, B. Laczy ${ }^{1}$, T. Kovács ${ }^{1}$, K. Böddi ${ }^{5}$, \\ A. Takátsy ${ }^{5}$, M. Gollasch ${ }^{2,3}$, A. Koller ${ }^{6,7,8}$, I. Wittmann \\ Affiliations \\ Affiliation addresses are listed at the end of the article
}

Key words
insulin
vasomotor function
ortho-tyrosine
eNOS

received 10.12 .2013

accepted 24.07.2014

Bibliography

DOI http://dx.doi.org/

10.1055/s-0034-1387701

Published online:

September 10, 2014

Horm Metab Res 2014;

46: 749-752

(c) Georg Thieme Verlag KG

Stuttgart · New York

ISSN 0018-5043

Correspondence

I. Wittmann, MD, PhD

$2^{\text {nd }}$ Department of Medicine and Nephrological Center

University of Pécs

1. Pacsirta Street

7624 Pécs

Hungary

Tel.: + 36/72/536050

Fax: + 36/72/536 051

istvan.wittmann@aok.pte.hu

\section{Luarastim}

\section{Abstract \\ $\nabla$}

Previous studies have shown that in diabetes mellitus, insulin-induced relaxation of arteries is impaired and the level of ortho-tyrosine (o-Tyr), an oxidized amino acid is increased. Thus, we hypothesized that elevated vascular level of 0 -Tyr contributes to the impairment of insulin-induced vascular relaxation. Rats were fed with 0 -Tyr for 4 weeks. Insulin-induced vasomotor responses of isolated femoral artery were studied using wire myography. Vascular o-Tyr content was measured by HPLC, whereas immunoblot analyses were preformed to detect eNOS phosphorylation. Sustained oral supplementation of rats with 0 -

\section{Introduction \\ $\nabla$}

Insulin is one of the major hormonal regulators of tissue metabolism, but it plays also an important role in regulating vasomotor activity $[1,2]$. Vascular effects of insulin could be manifested in dilation and/or constriction depending on tissues of origin [3]. The vasodilator action of insulin is primarily attributed to the release of nitric oxide (NO) produced by NO synthase in the endothelium (eNOS), which is activated via the phosphatidylinositol 3-kinase/Akt (PI3K/Akt) pathway [4-6].

In diabetes, increased levels of reactive oxygen species (ROS) play an important role in the development of impaired vasomotor response to insulin [7-9]. One target of the oxidative damage is proteins, which could lead to cellular dysfunction. In addition to direct damage by reactive oxidative metabolites, however, alternative pathomechanisms have been proposed, but they have received little attention. For example, superoxide can be metabolized to $\mathrm{H}_{2} \mathrm{O}_{2}$, which can give rise to the production of more reactive intermediates, such as hydroxyl radical $(\bullet \mathrm{OH})[10]$, which - among
Tyr increased the content of 0 -Tyr in the arterial wall and significantly reduced the relaxations to insulin. Sustained supplementation of cultured endothelial cells with 0 -Tyr increased the incorporation of $o$-Tyr and mitigated eNOS Ser (1177) phosphorylation to insulin. Increasing arterial wall $o$-Tyr level attenuates insulin-induced relaxation - at least in part - by decreasing eNOS activation. Elevated level of $o$-Tyr could be an underlying mechanism for vasomotor dysfunction in diabetes mellitus.

Supporting Information for this article is available online at http://www.thieme-connect. de/products

others - can modify phenylalanine residues to form para-, meta- and ortho-tyrosines ( $p-, m$ - and o-Tyr) [11-14]. Modified amino acids could originate from protein-bound amino acids [11] or may be incorporated into proteins during their synthesis resulting in a polypeptide without direct oxidative damage of the protein itself [15-17]. In line with these findings, free $m$-Tyr was shown to be incorporated into cellular proteins, possibly via protein synthesis, which then can exert cytotoxic actions [17]. Furthermore, $o$-Tyr and $m$-Tyr levels were found to be significantly higher in the aortic tissue of hyperglycemic cynomolgus monkeys and that of rats with aortic banding-induced hypertension $[11,18]$. Misincorporation of $o$-Tyr and $m$ Tyr into structural or catalytic proteins could also contribute to impaired cellular function, such as erythropoietin-hyporesponsiveness in erythroblasts and inhibition of tumor growth in vivo, possibly by interfering with MAP/ERK signaling $[19,20]$. Of note, cytotoxicity of $m$-Tyr can be blocked in vitro with phenylalanine [15], suggesting that L-phenylalanine tRNA synthase recognizes $m$-Tyr to affect incorporation of ROS-damaged amino acids into cellular proteins [15]. However, 

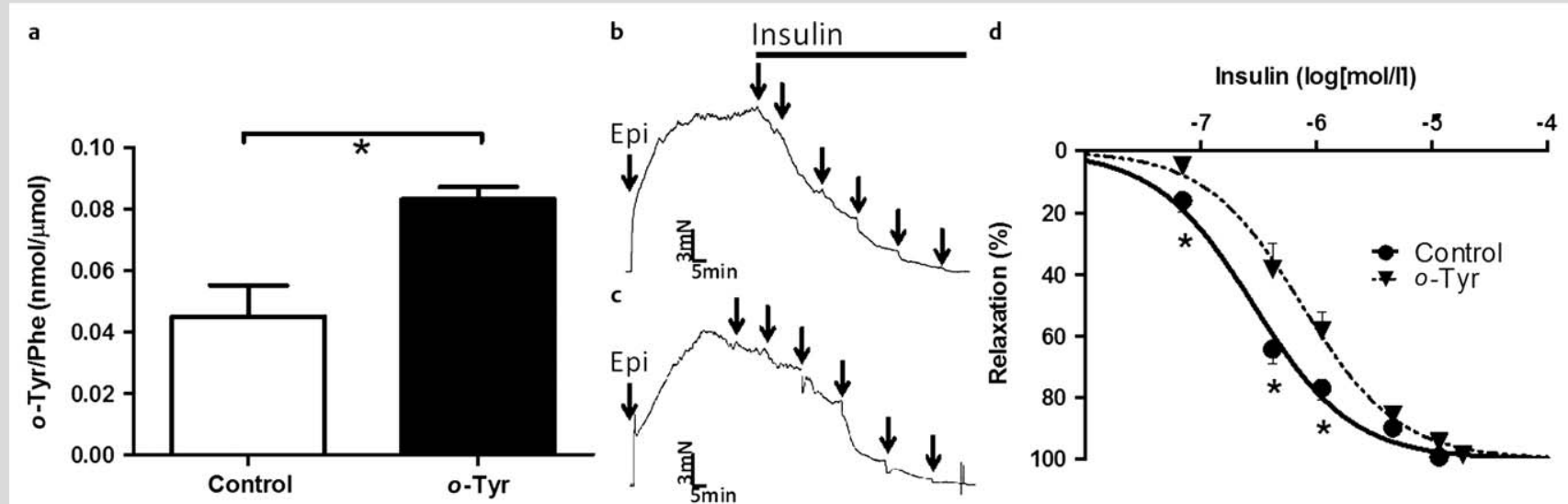

Fig. 1 a o-Tyr levels in the vascular wall of femoral arteries isolated from nonsupplemented and o-Tyr supplemented rats. There was a significantly increased $o$-Tyr level in the femoral artery isolated from the $o$-Tyr supplemented compared to the non-supplemented rats. $o$-Tyr levels are normalized to the phenylalanine (Phe) levels. Recordings of relaxation of femoral arteries isolated from $\mathbf{b}$ nonsupplemented, and $\mathbf{c}$ o-Tyr supplemented rats in response to increasing concentrations of insulin. $\mathbf{d}$ The dose-response curve of insulin-induced relaxations shows a rightward shift for femoral arteries of $o$-Tyr supplemented rats compared to femoral arteries of untreated control rats. Control $n=4 ; o-T y r n=4$. Data are means \pm SEM (Mann-Whitney test) ${ }^{*} p<0.05$.

incorporation of $m$-Tyr and $o$-Tyr into vascular proteins and the physiological consequences of exogenous administration of these tyrosine isomers on vasomotor function have not yet been investigated.

On the basis of the aforementioned findings and because tyrosine kinases are known to be also important in the activation of eNOS by phosphorylation of eNOS Ser (1177) [6] we hypothesized that accumulation of $o$-Tyr in the vascular wall can contribute to impaired relaxation of arteries to insulin by interfering with the PI3K/Akt/eNOS/NO signaling pathway. Thus, we determined the effects of oral supplementation of $o$-Tyr on the vasomotor responses of isolated arteries to insulin and investigated the phosphorylation of eNOS in response to $o$-Tyr and $p$-Tyr treatment in cultured endothelial cells.

\section{Material and Methods}

$\nabla$

Animals and experimental design

Animal experiments were carried out with the permission of the Animal Experiment Committee of the University of Pécs, Hungary. Male Sprague-Dawley rats (4-5 week-old; 90-120 g) were used in the tyrosine isomer incorporation studies. Rats were orally supplied with either $1.76 \mathrm{mg} /$ day of $o$-Tyr or vehicle during 6 days per week for 4 weeks. In general, there were no observable differences between the groups. At the end of 4 -week treatment, HPLC measurements and vasomotor studies were performed.

\section{Assessment of ortho-tyrosine and tyrosine isomer incorporation into arterial segments}

Proximal sections of the isolated femoral artery were hydrolyzed and then levels of phenylalanine and tyrosine isoforms were analyzed by HPLC using a fluorescent detector [13].

\section{Assessment of vasomotor function}

The modified method described by Fésüs et al. [21] was used. Femoral rings were pre-constricted with $100 \mathrm{nM}$ epinephrine. The magnitude of relaxation caused by insulin was expressed as the percentage of the isometric (Danish Multimyograph Model
$610 \mathrm{M})$ contraction evoked by epinephrine, which was taken as $100 \%$.

\section{Endothelial cell treatments}

Primary cultures of mouse endothelial cells (ECs) were randomly assigned into 2 groups and were incubated in media containing 1) $400 \mu \mathrm{M} p$-Tyr (Control) and 2) 400-400 $\mu \mathrm{M} p$-Tyr and $o$-Tyr for 8 days. Then the uptake of $o$-Tyr was analyzed. Prior to assessing eNOS phosphorylation, ECs were incubated with insulin ( $400 \mathrm{nM}$ for $5 \mathrm{~min}$ ) to activate endothelial nitric oxide synthase (eNOS) phosphorylation.

\section{Assessment of tyrosine isomer incorporation in endothelial cells}

The levels of protein-bound $o$-Tyr of ECs were analyzed by HPLC method as described by Molnár et al. [13].

\section{Immunoblot analyses}

Immunoblot analyses to detect eNOS phosphorylation were performed as we previously described [22]. Phospho-(Ser1177)eNOS levels were corrected for total eNOS.

\section{Statistical analyses}

Data are expressed as means \pm SEM. Statistical analyses were performed with unpaired t-test, and nonparametric tests as appropriate. Statistically significant differences were defined with $\mathrm{p} \leq 0.05$.

\section{Results}

$\nabla$

Effects of chronic oral o-Tyr supplementation of rats on 0 -Tyr levels in the vascular wall of isolated arterial segments

Compared to the control vessels of vehicle-treated rats, vascular $o$-Tyr content significant increased as a result of 4 weeks of oral supplementation of rats with 0 -Tyr $(\bullet$ Fig. 1a). 


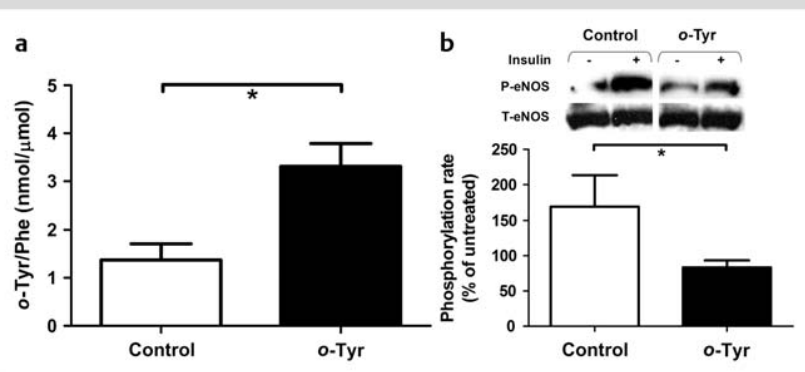

Fig. 2 a Incorporation of o-Tyr into proteins of cultured endothelial cells. Protein-bound 0 -Tyr levels are shown after 8 days of incubation of endothelial cells with normal medium or o-Tyr supplemented media. As a result of $o$-Tyr treatment cellular incorporation significantly increased. Cellular 0 -Tyr levels are expressed relative to phenylalanine (Phe) levels (Control $n=7 ; 0$-Tyr $n=7$ ). b Effect of $o$-Tyr administration on insulininduced eNOS phosphorylation in cultured endothelial cells. Upper panel: Immunoblots showing changes in eNOS Ser (1177) phosphorylation (P-eNOS) in untreated and insulin-treated $(400 \mathrm{nM})$ endothelial cells cultured in normal medium (Control) or o-Tyr supplemented media. Lower panel: Densitometry shows that insulin-induced eNOS phosphorylation was significantly $\left({ }^{*} p<0.05\right)$ reduced in $o$-Tyr-cultured cells compared to untreated control cells (Control). The phospho-eNOS level is expressed as the percentage of insulin-untreated cells after normalization to total eNOS (T-eNOS). Control $n=5 ; 0-T y r n=4$. Data are means \pm SEM (a MannWhitney test; $\mathbf{b}$ Unpaired $t$-test). Data are means \pm SEM (a Unpaired t-test; b Mann-Whitney test). ${ }^{*} \mathrm{p}<0.05$.

\section{Effects of chronic 0 -Tyr supplementation of rats on} insulin-induced relaxation of isolated femoral arteries Original recordings of isometric tension of femoral arteries of control (nonsupplemented) rat show that insulin elicited substantial relaxation ( $\boldsymbol{O}$ Fig. $\mathbf{1 b}$ ), whereas a vessel of 0 -Tyr-treated rats exhibited reduced relaxation $(\bullet$ Fig. 1 c). Summary data show a significantly diminished relaxation of the femoral artery of $o$-Tyr-treated rats in response to insulin compared to arteries of vehicle-treated rats ( $\bullet$ Fig. 1 d).

\section{Incorporation of $\mathrm{o}$-Tyr into proteins of cultured endothelial cells}

To investigate the incorporation of $o$-Tyr into endothelial proteins, we measured the relative concentration of protein-bound $o$-Tyr levels in endothelial cells (ECs) cultured in control conditions and $o$-Tyr supplemented media for 8 days. Proteins from $o$-Tyr-cultured ECs showed a higher 0 -Tyr content compared to control ECs ( $\bullet$ Fig. 2a).

\section{Effects of $o$-Tyr on insulin-induced eNOS}

\section{phosphorylation in cultured endothelial cells}

Immunoblot analyses show that activating eNOS phosphorylation [eNOS Ser (1177)] in response to insulin was significantly reduced in ECs cultured in the presence of $o$-Tyr compared to untreated control cells ( $\bullet$ Fig. 2 b).

\section{Discussion}

$\nabla$

In the present study, we have shown for the first time that vasoincorporation of $o$-Tyr reduces arterial relaxation to insulin and decreases eNOS phosphorylation in ECs. We interpret these finding to mean that increased levels of vascular 0 -Tyr inhibit eNOS phosphorylation and thus reduced synthesis of NO can result in impaired relaxation to insulin.
Previous studies have shown that 0 -Tyr levels are increased in aortic tissues in diabetes mellitus [11,23], which raised the possibility that $o$-Tyr content contributes to insulin-induced vasomotor dysfunction. However, diabetes is a complex disease and it is likely that many pathomechanisms are activated simultaneously. In order to elucidate the potential role of elevated cellular level of $o$-Tyr in modulation of insulin-induced arterial relaxation we used a model of chronic oral administration of $o$-Tyr. We have found that sustained oral treatment of rats with $o$-Tyr markedly increased vascular content of $o$-Tyr and at the same time impaired the insulin-induced relaxation in isolated femoral arteries.

The findings that vascular 0 -Tyr content modulates the magnitude of vasomotor responses to insulin and that increasing 0 -Tyr content attenuates insulin-induced vasorelaxations suggest a novel pathophysiological mechanism, which could be important in the development of not only vasomotor dysfunction, but in general, macro- and microangiopathy known to be present in diabetes mellitus. Moreover, increased level of 0 -Tyr due to exogenous (e.g., nutrition) $[24,25]$ or endogenous sources other than diabetes mellitus, such as hypertension [18] or inflammation [26] (known to be associated with oxidative stress) could promote $o$-Tyr incorporation into vascular wall and impair vasomotor function.

It is known that insulin-induced relaxation is primarily mediated by eNOS derived NO (Supplementary Fig. 1S) [4-6]. Thus, to elucidate possible underlying mechanisms responsible for the reduced arterial relaxation to insulin, we cultured endothelial cells and investigated the effects of $o$-Tyr treatment on eNOS phosphorylation. We found that protein-bound $o$-Tyr levels significantly increased in $o$-Tyr-cultured endothelial cells, which was accompanied by attenuated eNOS phosphorylation in response to insulin. These findings suggest that incorporation of $o$-Tyr impairs eNOS function, that is, synthesis of NO, which could explain how 0 -Tyr interferes with NO mediated relaxation to insulin. On the basis of the findings of the present and previous studies, we also propose that deterioration of the PI3K/Akt/ eNOS/NO pathway that mediates the vasorelaxation to insulin could contribute to development of vascular insulin resistance $[4,5]$.

\section{Clinical significance}

Impaired regulation of tissue blood flow in diabetes mellitus and its consequences are major clinical problems being one of the major health burdens world-wide $[1,2]$. Thus, understanding the molecular pathomechanisms of vascular insulin resistance can have tremendous beneficial implications for interfering and treating diabetes related vasomotor dysfunction, such as macroand micorangiopathies. Specifically, our study suggests that accumulation of $o$-Tyr in the vascular wall may contribute to the impaired insulin-induced relaxation by interfering with the production of vasodilator NO by eNOS. Patients with type 2 diabetes and chronic kidney disease (CKD) have an increased $o$-Tyr burden [13,14,23,27], which may contribute to the development of vascular complications. Interfering with this pathway could represent a promising future strategy for the prevention and/or treatment of vascular complications in diabetes mellitus.

\section{Limitations and future perspectives}

Although, the present study demonstrates that chronic oral supplementation of $o$-Tyr can increase levels of $o$-Tyr in arterial wall tissue with functional consequences of impaired vasomotor 
responses to insulin, the detailed molecular signaling pathways in the vasculature need to be elucidated by further studies.

\section{Acknowledgements}

The work was supported by the American Heart Association, Founders Affiliate, 0855910D, Hungarian National Science Research Fund (OTKA) K 108444, SROP-4.2.2.A-11/1/KONV2012-0024, SROP-4.2.2.A-11/1/KONV-2012-0017 (to AK) and by the European Union and the State of Hungary, co-financed by the European Social Fund in the framework of TÁMOP-4.2.4.A/ 2-11/1-2012-0001 'National Excellence Program' (to GAM). This work was also supported by the DAAD. We express our sincere thanks to Dr. Ilona Sámikné Varga and Ildikó Fábián for their excellent technical assistance.

\section{Conflict of Interest}

The authors declare that there is no duality of interest associated with this manuscript.

\section{Affiliations}

${ }^{1}{ }^{\text {nd }}$ Department of Medicine and Nephrological Center, University of Pécs, Pécs, Hungary

Experimental and Clinical Research Center (ECRC), Max Delbrück Center for Molecular Medicine, Berlin, Germany

${ }^{3}$ Medical Clinic for Nephrology and Internal Intensive Care, Charité Campus Virchow Klinikum, Berlin, Germany

${ }^{4}$ Interdisziplinäres Stoffwechsel-Centrum, Charité, Campus Virchow Klinikum, Berlin, Germany

5 Department of Biochemistry and Medical Chemistry, University of Pécs,

Pécs, Hungary

${ }^{6}$ Department of Pathophysiology and Gerontology and Szentágothai Research Centre University of Pécs, Hungary

Department of Physiology, New York Medical College, Valhalla, NY, USA

${ }^{8}$ Department of Pathophysiology, Semmelweis University, Budapest, Hungary

\section{References}

1 Saltiel AR, Kahn CR. Insulin signalling and the regulation of glucose and lipid metabolism. Nature 2001; 414: 799-806

2 Muniyappa R, Montagnani M, Koh KK, Quon MJ. Cardiovascular actions of insulin. Endocr Rev 2007; 28: 463-491

3 Eringa EC, Stehouwer CD, van Nieuw Amerongen GP, Ouwehand L, Westerhof $N$, Sipkema P. Vasoconstrictor effects of insulin in skeletal muscle arterioles are mediated by ERK1/2 activation in endothelium. Am J Physiol Heart Circ Physiol 2004; 287: H2043-H2048

4 Steinberg HO, Brechtel G, Johnson A, Fineberg N, Baron AD. Insulinmediated skeletal muscle vasodilation is nitric oxide dependent. A novel action of insulin to increase nitric oxide release. J Clin Invest 1994; 94: 1172-1179

5 Lee JH, Ragolia L.AKT. AKT phosphorylation is essential for insulininduced relaxation of rat vascular smooth muscle cells. Am J Physiol Cell Physiol 2006; 291: C1355-C1365

6 Forstermann U, Sessa WC. Nitric oxide synthases: regulation and function. European heart journal 2012; 33: 829-837, 837a-837d

7 Houstis $N$, Rosen ED, Lander ES. Reactive oxygen species have a causal role in multiple forms of insulin resistance. Nature 2006; 440: 944-948

8 Bagi Z, Koller A, Kaley G. Superoxide-NO interaction decreases flowand agonist-induced dilations of coronary arterioles in Type 2 diabetes mellitus. Am J Physiol Heart Circ Physiol 2003; 285: H1404-H1410
9 Bagi Z, Koller A. Lack of nitric oxide mediation of flow-dependent arteriolar dilation in type I diabetes is restored by sepiapterin. J Vasc Res 2003; 40: 47-57

10 Pieper GM, Langenstroer P, Siebeneich W. Diabetic-induced endothelial dysfunction in rat aorta: role of hydroxyl radicals. Cardiovasc Res 1997; 34: 145-156

11 Pennathur S, Wagner JD, Leeuwenburgh C, Litwak KN, Heinecke JW. A hydroxyl radical-like species oxidizes cynomolgus monkey artery wall proteins in early diabetic vascular disease. J Clin Invest 2001; 107: 853-860

12 Biondi R, Ambrosio G, Liebgott T, Cardounel AJ, Bettini M, Tritto I, Zweier $J L$. Hydroxylation of D-phenylalanine as a novel approach to detect hydroxyl radicals: application to cardiac pathophysiology. Cardiovasc Res 2006; 71: 322-330

13 Molnar GA, Nemes V, Biro Z, Ludany A, Wagner Z, Wittmann I. Accumulation of the hydroxyl free radical markers meta-, ortho-tyrosine and DOPA in cataractous lenses is accompanied by a lower protein and phenylalanine content of the water-soluble phase. Free Radic Res 2005; 39: 1359-1366

14 Molnar GA, Wagner Z, Marko L, Ko Szegi T, Mohas M, Kocsis B, Matus Z, Wagner L, Tamasko M, Mazak I, Laczy B, Nagy J, Wittmann I. Urinary ortho-tyrosine excretion in diabetes mellitus and renal failure: evidence for hydroxyl radical production. Kidney Int 2005; 68: 2281-2287

15 Gurer-Orhan H, Ercal N, Mare S, Pennathur S, Orhan H, Heinecke JW. Misincorporation of free m-tyrosine into cellular proteins: a potential cytotoxic mechanism for oxidized amino acids. Biochem J 2006; 395: 277-284

16 Bertin C, Weston LA, Huang T, Jander G, Owens T, Meinwald J, Schroeder $F C$. Grass roots chemistry: meta-tyrosine, an herbicidal nonprotein amino acid. Proc Natl Acad Sci USA 2007; 104: 16964-16969

17 Klipcan L, Moor N, Kessler N, Safro MG. Eukaryotic cytosolic and mitochondrial phenylalanyl-tRNA synthetases catalyze the charging of tRNA with the meta-tyrosine. Proc Natl Acad Sci USA 2009; 106: 11045-11048

18 Szijarto IA, Molnar GA, Mikolas E, Fisi V, Laczy B, Gollasch M, Koller $A$, Wittmann I. Increase in insulin-induced relaxation of consecutive arterial segments toward the periphery: Role of vascular oxidative state. Free Radic Res 2014; 48: 749-757

19 Mikolas E, Kun S, Laczy B, Molnar GA, Selley E, Koszegi T, Wittmann I. Incorporation of ortho- and meta-tyrosine into cellular proteins leads to erythropoietin-resistance in an erythroid cell line. Kidney Blood Press Res 2013; 38: 217-225

20 Ruggiero RA, Bruzzo J, Chiarella P, Bustuoabad OD, Meiss RP, Pasqualin $C D$. Concomitant tumor resistance: the role of tyrosine isomers in the mechanisms of metastases control. Cancer Res 2012; 72: 1043-1050

21 Fesus G, Dubrovska G, Gorzelniak K, Kluge R, Huang Y, Luft FC, Gollasch M. Adiponectin is a novel humoral vasodilator. Cardiovasc Res 2007; 75: 719-727

22 Wagner L, Laczy B, Tamasko M, Mazak I, Marko L, Molnar GA, Wagner Z, Mohas M, Cseh J, Fekete A, Wittmann I. Cigarette smoke-induced alterations in endothelial nitric oxide synthase phosphorylation: role of protein kinase C. Endothelium 2007; 14: 245-255

23 Vivekanadan-Giri A, Wang JH, Byun J, Pennathur S. Mass spectrometric quantification of amino acid oxidation products identifies oxidative mechanisms of diabetic end-organ damage. Rev Endocr Metab Disord 2008; 9: 275-287

24 Karam LR, Simic MG. Formation of ortho-tyrosine by radiation and organic solvents in chicken tissue. J Biol Chem 1990; 265: 11581-11585

25 Ledo A, Arduini A, Asensi MA, Sastre J, Escrig R, Brugada M, Aguar M, Saenz $P$, Vento $M$. Human milk enhances antioxidant defenses against hydroxyl radical aggression in preterm infants. Am J Clin Nutr 2009; 89: $210-215$

26 Onorato JM, Thorpe SR, Baynes JW. Immunohistochemical and ELISA assays for biomarkers of oxidative stress in aging and disease. Annals of the New York Academy of Sciences 1998; 854: 277-290

27 Kun S, Mikolas E, Molnar GA, Selley E, Laczy B, Csiky B, Kovacs T, Wittmann I. Association of plasma ortho-tyrosine/para-tyrosine ratio with responsiveness of erythropoiesis-stimulating agent in dialyzed patients. Redox Rep 2014; 19: 190-198 